\title{
A NEW PROOF OF BROWN'S COLLARING THEOREM
}

\section{ROBERT CONNELLY}

The aim of this note is to give a new proof that if a subspace $B$, compact for convenience, is locally collared in a space $X$, then it is collared. The idea of the proof is simply to add a collar $B \times I$ to $X$ to get $X^{+}$and then to construct a homeomorphism of $X$ with $X^{+}$by pushing $B$ down on one collared open set at a time.

The theorem, of course, is essentially that of [1]. However, the proof easily works in the piecewise linear (PL) category (i.e. all maps are $\mathrm{PL}$ and spaces are polyhedra), and when $B$, the boundary, is a pair or flag, cf. [3]. At the end of the paper we shall note briefly how the noncompact case and the PL case can be handled by our techniques.

A closed subspace $B C X$ is locally collared if $B$ is covered by sets $U$, open in $B$, such that for each $U$ there is a closed embedding $h: \bar{U} \times[0,1] \rightarrow X$ such that $h^{-1}(B)=\bar{U} \times\{0\}, h(x, 0)=x$ for $x \in \bar{U}$, and $h(U \times[0,1))$ is open in $X$. For metric spaces this is equivalent to the definition in [1]. $B$ is said to be collared if one $U$ can be taken to be all of $B$.

THEOREM. If $B C X$ is compact and locally collared in $X$, which is Hausdorff, then $B$ is collared in $X$.

Proof. Let $U_{1}, U_{2}, \cdots, U_{n}$ be an open cover of $B$ such that each $U_{i}$ is as in the definition. By the normality of $B$, shrink the cover to find another cover $V_{1}, \cdots, V_{n}$ such that $\bar{V}_{i} \subset U_{i}, i=1, \cdots, n$. Let $X^{+}=X \cup B \times[-1,0]$ where $(x, 0)$ is identified to $x$, and let $h_{i}: \vec{U}_{i}$ $\times[0,1] \rightarrow X$ be the embeddings given by the local collars. Let $H_{i}: \vec{U}_{i} \times[-1,1] \rightarrow X^{+}, i=1, \cdots, n$, be the embedding defined by

$$
\begin{aligned}
H_{i}(x) & =h_{i}(x) & & \text { for } x \in \bar{U}_{i} \times[0,1], \\
& =x & & \text { for } x \in \bar{U}_{i} \times[-1,0] .
\end{aligned}
$$

Inductively we shall define maps $f_{i}: B \rightarrow[-1,0]$ and embeddings $g_{i}: X \rightarrow X^{+}, i=0,1, \cdots, n$, such that

Received by the editors May 22, 1969.

AMS subject classifications. Primary 5701.

Copyright (c) 1971, American Mathematical Society 
(a) $f_{i}(x)=-1$ if $x \in \bigcup_{j \leq i} \bar{V}_{j}$,

(b) $g_{i}(x)=\left(x, f_{i}(x)\right)$ if $x \in B$, and

(c) $g_{i}(X)=X \cup\left\{(x, t) \mid t \geqq f_{i}(x)\right\}$.

Note that since the $V_{i}$ 's cover $B, g_{n}(X)=X^{+}$and thus $g_{n}^{-1}$ will give the required collar.

Define $g_{0}=1$, and inductively suppose $g_{i-1}$ has been defined. Let $\phi_{i}: H_{i}^{-1} g_{i-1}(X) \rightarrow \bar{U}_{i} \times[-1,1]$ be an embedding that pushes down along fibers such that $\phi_{i} H_{i}^{-1} g_{i-1}\left(\bar{V}_{i}\right)=\bar{V}_{i} \times\{-1\}$ and $\phi_{i} \mid\left(\bar{U}_{i}-U_{i}\right)$ $\times[-1,1] \cup \bar{U}_{i} \times\{1\}=1$. Such a $\phi_{i}$ can be defined as follows: Let $\lambda_{i}: \bar{U}_{i} \rightarrow[0,1]$ be a Urysohn function which is 0 on $\bar{U}_{i}-U_{i}$ and 1 on $\bar{V}_{i}$. Let $s_{x}:\left[f_{i-1}(x), 1\right] \rightarrow\left[\left(1-\lambda_{i}(x)\right) f_{i-1}(x)+\lambda_{i}(x)(-1), 1\right]$ be the unique order preserving simplicial homeomorphism given by $s_{x}(t)$ $=((b-1) /(a-1))(t-1)+1$ where $a=f_{i-1}(x)$ and $b=\left(1-\lambda_{i}(x)\right) f_{i-1}(x)$ $+\lambda_{i}(x)(-1)$. Now define $\phi_{i}(x, t)=\left(x, s_{x}(t)\right)$. Clearly $\phi_{i}$ is continuous. Then define $\Phi_{i}: g_{i-1}(X) \rightarrow X^{+}$by:

$$
\begin{aligned}
\Phi_{i}(x) & =H_{i} \phi_{i} H_{i}^{-1}(x) & & \text { for } x \in g_{i-1}(X) \cap H_{i}(\bar{U} \times[-1,1]), \\
& =x & & \text { otherwise, }
\end{aligned}
$$

and $g_{i}=\Phi_{i} g_{i-1}$. Clearly $\Phi_{i}$ and thus $g_{i}$ is well defined and an embedding since $\phi_{i} \mid\left(\bar{U}_{i}-U_{i}\right) \times[-1,1] \cup \bar{U}_{i} \times\{1\}=1, \phi_{i}$ is an embedding (since each $s_{x}$ is), and $g_{i-1}(X) \cap H_{i}\left(\bar{U}_{i} \times[-1,1]\right)=H_{i}\left(\bar{U}_{i} \times[0,1]\right.$ ) $\cup\left\{(x, t) \mid t \geqq f_{i-1}(x)\right.$ and $\left.x \in \bar{U}_{i}\right\}$ by (c) for $g_{i-1}$. Note that (b) now defines $f_{i}(x)$, and (a) and (c) are satisfied by construction.

Remark 1. The noncompact case. The method of proof used above can be extended to certain cases when $X$ is not compact. For instance, the proof works if we assume that $X$ has a slightly stronger property than paracompactness, namely if every open cover has a star finite refinement (cf. [2] for definitions). In this case it is possible to order the $U_{i}$ 's, although infinite, so that every point in $X^{+}$has an open neighborhood which moves only finitely of ten.

REMARK 2. The PL case. The theorem is still true if all spaces and maps mentioned (including the definition of local collaring) are polyhedra and PL respectively. The same proof goes through except that the particular definition of $\phi_{i}$ must be altered slightly. Namely to make $\phi_{i} \mathrm{PL}$ it is easiest to triangulate $\bar{U}_{i} \times[-1,1]$ so that $\bar{V}_{i}$ $\times[-1,1]$ and $H_{i}{ }^{-1} g_{i-1}(X)$ are subcomplexes and projection $\pi: \bar{U}_{i}$ $\times[-1,1] \rightarrow \bar{U}_{i}$ is simplicial. Then it is easy to define a simplicial map $\phi_{i}$ so that it has the desired properties.

The author wishes to thank the referee for many useful comments and suggestions. 


\section{REFERENCES}

1. Morton Brown, Locally flat embeddings of topological manifolds, Topology of 3Manifolds and Related Topics (Proc. the Univ. of Georgia Inst., 1961), Prentice-Hall, Englewood Cliffs, N. J., 1962, pp. 83-91. MR 28 \#1598.

2. James Dugundji, Topology, Allyn and Bacon, Boston, Mass., 1966. MR 33 $\# 1824$.

3. C. P. Rourke, Covering the track of an isotopy, Proc. Amer. Math. Soc. 18 (1967), 320-324. MR 36 \#7145.

Cornell University, Ithaca, New York 14850

University of Michigan, ANn Arbor, Michigan 48104 